\title{
Breakdown of quantum-to-classical correspondence for diffusion in a high-temperature thermal environment
}

\author{
Dekel Shapira and Doron Cohen 10 \\ Department of Physics, Ben-Gurion University of the Negev, Beer-Sheva 84105, Israel
}

(Received 21 July 2020; revised 29 October 2020; accepted 12 January 2021; published 12 February 2021)

\begin{abstract}
We reconsider the old problem of Brownian motion in a homogeneous high-temperature thermal environment. The semiclassical theory implies that the diffusion coefficient does not depend on whether the thermal fluctuations are correlated in space or disordered. We show that the corresponding quantum analysis exhibits a remarkable breakdown of quantum-to-classical correspondence. Explicit results are found for a tight-binding model, within the framework of an Ohmic master equation, where we distinguish between on-site and on-bond dissipators. The breakdown is second order in the inverse temperature and therefore, on the quantitative side, involves an inherent ambiguity that is related to the Ohmic approximation scheme.
\end{abstract}

DOI: 10.1103/PhysRevResearch.3.013141

\section{INTRODUCTION}

The fingerprints of quantum mechanics on Brownian motion is an intriguing theme [1-3]. This theme concerns also the motion of a particle or an exciton on a lattice [4-18], or the closely related studies of motion in a washboard potential [19-21].

The traditional paradigm is/was that the effects of quantum mechanics show up only at low temperatures, where nonclassical effects are related to the failure of the Markovian approximation. This view has been challenged by publications regarding excitation transport in photosynthetic light-harvesting complexes, most notably by the experiment in [22], and by many theoretical publications [23-32]. But by now it has been argued [33-38] that the transport there, by itself, is "classical" in nature.

Nevertheless, contrary to the traditional paradigm, we suggest below that quantum manifestation in stochastic motion can be detected via the high-temperature dependence of the transport coefficients. This opens an avenue for challenging the traditional (classical) paradigm of Brownian motion.

\section{A. Brownian motion}

High-temperature $(T)$ classical Brownian motion is described by the Langevin equation

$$
\dot{p}=-\eta \dot{x}+f,
$$

where $f$ is the white noise of intensity $v$, related to the friction coefficient via the fluctuation dissipation relation

$$
v=2 \eta T . \quad \text { [can be used as definition of } T \text { ] }
$$

Published by the American Physical Society under the terms of the Creative Commons Attribution 4.0 International license. Further distribution of this work must maintain attribution to the author(s) and the published article's title, journal citation, and DOI.
For the standard dispersion relation $\dot{x}=(1 / \mathrm{m}) p$, where $\mathrm{m}$ is the mass of the particle, one obtains the following simple results for the transport coefficients:

$$
\begin{gathered}
\mu=\frac{1}{\eta}, \quad \text { [mobility] } \\
\left.D=\frac{T}{\eta} . \quad \text { [diffusion coefficient }\right]
\end{gathered}
$$

The mobility $\mu$ is used to determine the drift velocity due to an applied bias, while $D$ is the coefficient that enters Fick's law. The Einstein relation $D / \mu=T$ is satisfied. It is important to realize that Eq. (2) characterizes the thermal environment, while the Einstein relation characterizes the dissipative dynamics of the particle.

\section{B. Quantum signature}

One wonders whether the dependence of the transport coefficients $(\mu$ and $D)$ on the dissipation parameters ( $\eta$ and $\nu$ ) is universal. This is the main question that motivates the present study.

\section{Common wisdom}

The high-temperature noise arises from a fluctuating potential, namely,

$$
f=-\partial_{x} \mathcal{U}(x, t) .
$$

This potential features in general a spatial correlation scale $\ell$. Semiclassically, the transport coefficients do not depend on $\ell$, and the common practice, as in the Caldeira-Leggett model [1,2], is to assume that $f$ is independent of $x$, meaning that $\ell=\infty$. But in the quantum treatment $\ell$ does show up in the analysis, because it determines the line shape of the stochastic kernel $\mathcal{W}\left(k \mid k^{\prime}\right)$ for scattering from momentum $k^{\prime}$ to momentum $k$. Namely, the width of the kernel $(\sim 2 \pi / \ell)$ has implications on the transient decoherence process [39-41]. Yet, one does not expect that this line shape will have any effect on the long-time spreading. The argument is simple: on 
the basis of the central limit theorem successive convolutions should lead to a result that does not depend on the $\ell$-dependent line shape of the stochastic kernel but only on its second moment, which is characterized by $v$. Consequently, robust quantum-to-classical correspondence (QCC) is expected at high temperatures. Such QCC can be regarded as an implication of the Thomas-Reiche-Kuhn-Bethe-Wang sum rule [42], or as an extension of the restricted QCC principle $[43,44]$.

Main statement. In the present work we show that $\ell$ independence of the transport coefficients $(\mu$ and $D)$ is a fallacy. Given $\eta$, we shall see that $D$ acquires a nonuniversal dependence on the temperature, which constitutes a quantummechanical signature.

\section{Tight-binding model}

Here we consider a particle or a single exciton that can hop along a one-dimensional chain whose sites are labeled by an integer index $x$. The dynamics of the isolated system is determined by the Hamiltonian

$$
\boldsymbol{H}^{(c)}=-c \cos (a \boldsymbol{p})-f_{0} \boldsymbol{x},
$$

where $a$ is the lattice constant, $c$ is the hopping frequency, and $f_{0}$ is an applied bias. The operators $e^{\text {Fiap }}$ generate one-site displacements. This Hamiltonian is of quantum-mechanical origin but may be treated semiclassically by deriving the equations of motion $\dot{\boldsymbol{x}}=c a \sin (a \boldsymbol{p})$ and $\dot{\boldsymbol{p}}=f_{0}$. Adopting the standard jargon of condensed matter textbooks, we shall call this semiclassical treatment of the dynamics. The exact quantum dynamics of Eq. (6) is obtained from the Schrodinger equation [45], or equivalently, from the Liouville-von Neumann equation for the probability matrix $\rho$.

The dynamics on the lattice features the dispersion relation $\dot{\boldsymbol{x}}=v(\boldsymbol{p})$, where $v(p)=c a \sin (a p)$. The continuum limit (small $a p$ ) leads to the standard dispersion relation $v=(1 / \mathrm{m}) p$ with $\mathrm{m}=1 /\left(c a^{2}\right)$. Therefore we can regard the latter case as a special regime of the former. Irrespective of the dispersion relation, if the particle is coupled to a thermal environment, the semiclassical treatment leads to $\dot{\boldsymbol{p}}=F(t)$, where the force contains a stochastic noise term and a friction term, namely, $F(t)=f_{0}+f(t)-\eta \dot{x}$. In the absence of external bias $\left(f_{0}=0\right)$, this leads to the Langevin equation, Eq. (1).

In the corresponding high-temperature Markovian quantum treatment, the dynamics is given by a master equation for the probability matrix [46]. This master equation incorporates an extra term, aka dissipator, that represents the noise and the friction:

$$
\frac{d \rho}{d t}=\mathcal{L} \rho=-i\left[\boldsymbol{H}^{(c)}, \rho\right]+\mathcal{L}^{(\text {bath })} \rho .
$$

The dissipator $\mathcal{L}^{\text {(bath) }}$ is determined by the coupling between the isolated chain and the environment, and depends on the temperature of the bath.

\section{Regime diagram}

Disregarding the optional applied bias $f_{0}$, the isolated tightbinding model has no free parameters (formally we can set the units of time and length such that $c=a=1$ ). With bath, the continuum version of quantum Brownian motion (QBM) features a single dimensionless parameter, the scaled inverse (a)

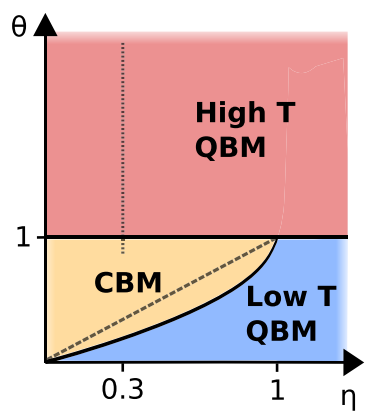

(b)

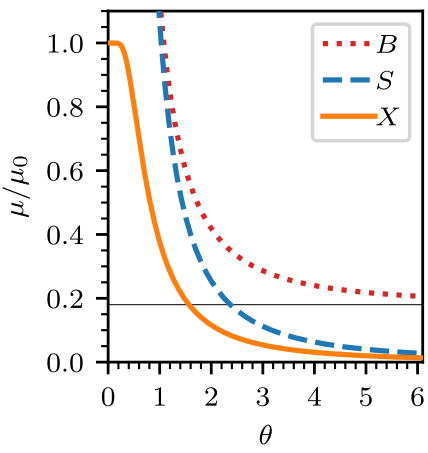

FIG. 1. The Brownian motion regime diagram. (a) The various regions in the $(\eta, \theta)$ diagram are indicated. We distinguish between the classical-like Brownian motion (CBM) region, the lowtemperature QBM region where memory effects dominate, and the high-temperature QBM region that is discussed in this work. Note that below the dashed diagonal line $(\beta>1)$ memory effects should be taken into account. (b) The scaled mobility $\mu / \mu_{0}$ where $\mu_{0}=1 / \eta$ vs $\theta$, based on the analytical results that have been obtained for diffusion in the $\mathrm{X} / \mathrm{S}$ coupling schemes. The result is independent of $\eta$. We also add the result for the $\mathrm{B}$ coupling scheme that approaches the finite asymptotic value $\mu_{\infty}=2 \eta$ (horizontal line). In the latter case $\eta=0.3$ has been assumed. Note that the $\mathrm{S} / \mathrm{B}$ results are applicable only in the $\theta>1$ regime.

temperature $\beta$, which is the ratio between the thermal time $1 / T$ and damping time $\mathrm{m} / \eta$. In the lattice problem one can define two dimensionless parameters

$$
\alpha=\frac{\eta a^{2}}{2 \pi}, \quad \theta=\frac{T}{c} .
$$

Accordingly, $\beta=\alpha / \theta$. In our model we set the units such that $a=1$, hence disregarding the $2 \pi$ factor, our scaled friction parameter $\eta$ is the same as $\alpha$. The regime diagram of the problem is displayed in Fig. 1 and is further discussed below. It contains both the classical-like Brownian motion (CBM) regime, where memory effects are either not expressed or appear as a transient, and QBM regimes where the dynamics is drastically different.

\section{E. Relation to past studies}

The standard analysis of QBM [3] reveals that quantumimplied memory effects are expressed in the regime $\beta>1$, where a transient $\log (t)$ spreading is observed in the absence of bias, followed by diffusion. The later quantum dissipation literature regarding the two-site spin-boson model [47] and regarding multisite chains $[7,21]$ is focused in this low-temperature regime where a transition from CBM-like behavior to overdamped or localized behavior is observed, notably for large $\alpha$ of order unity.

Our interest is focused in the $\alpha, \beta \ll 1$ regime. This regime is roughly divided into two regions by the line $\theta \sim 1$, see Fig. 1. Along this line the thermal de Broglie wavelength of the particle is of the order of the lattice constant, and hence it bears formal analogy to the analysis of QBM in the cosine potential [20], where it marks the border to the regime where the activation mechanism comes into action. 
In our tight-binding model we have a single band, and hence transport via thermal activation is not possible. Rather, in the $\theta>1$ regime the momentum distribution within the band is roughly flat. To avoid misunderstanding, what we call in the present study the "high-temperature" regime assumes a single band approximation by construction.

\section{F. Outline}

An overview of the main results is presented in Sec. II. The Ohmic master equation is explained in Sec. III. The semiclassical analysis is detailed in Secs. IV-VI. The quantum analysis is detailed in Sec. VII. The effective stochastic description is presented in Sec. VIII, where we discuss detailed-balance consideration as well. A concise summary is provided in Sec. IX.

\section{OVERVIEW OF MAIN RESULTS}

In order to demonstrate that the temperature dependency of the transport coefficients is $\ell$ dependent, we consider in detail two extreme cases: (a) The Caldeira-Leggett X dissipator $\mathcal{L}^{(\mathrm{X})}$ where a single bath is coupled to $\boldsymbol{x}$. This corresponds to a nondisordered $(\ell=\infty)$ bath. (b) The $S$ dissipator $\mathcal{L}^{(S)}$, where each site is coupled to an independent bath. For this coupling $\ell=a$. In Sec. VIII we also present results for intermediate values of $\ell$. For completeness we also consider another case: (c) the $\mathrm{B}$ dissipator $\mathcal{L}^{(\mathrm{B})}$, where each bond is coupled to an independent bath. For all cases the dynamics is governed by the Ohmic master equation Eq. (7), and the dissipator $\mathcal{L}^{\text {(bath) }}$ takes different forms according to the couplings. For the three cases above, the bath parameters are $\nu_{i}$ and $\eta_{i}$ with $i=X, S, B$.

\section{A. $\mathrm{X}$ dissipation}

As a reference case we calculate the transport coefficients for a particle in a tight-binding model that is coupled to an Ohmic Caldeira-Leggett bath via the $x$ variable. We term this standard case "X dissipation." We set the length units such that $a=1$. The bath parameters $v_{X}$ and $\eta_{X}$ are chosen such that in the semiclassical Eq. (1) we have $v=v_{X}$ and $\eta=\eta_{X}$. The result that we get for the diffusion coefficient is

$$
D^{(\mathrm{X})}=\left[1-\frac{1}{\left[\mathrm{I}_{0}(c / T)\right]^{2}}\right] \frac{T}{\eta},
$$

where $\mathrm{I}_{n}$ is the modified Bessel function. This result is exact to the extent that the (Markovian) Ohmic master equation can be trusted. For the mobility we get $\mu=D / T$, as expected for the Einstein relation. A plot of the mobility versus temperature is provided in Fig. 1.

For low temperatures (in the sense $T \ll c$ ) one recovers the standard results Eqs. (3) and (4) that apply for nonrelativistic (linear) dispersion. For high temperatures the result takes the form $D^{(\mathrm{X})}=D_{\|}$, with

$$
D_{\|} \approx C_{\|}\left[1+A_{\|}\left(\frac{c}{T}\right)^{2}\right] \frac{c^{2}}{v},
$$

where $C_{\|}=1$ and $A_{\|}=-5 / 16$. The reason for using the subscript notation is clarified below. The same expression appears for the $\mathrm{S} / \mathrm{B}$ dissipators, with $v$ replaced by $v_{S}$ and

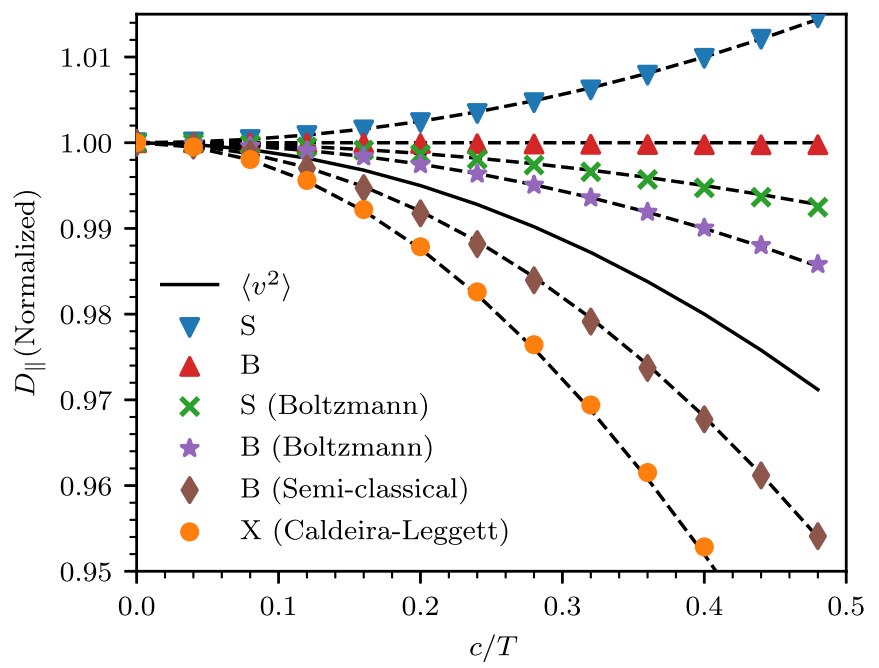

FIG. 2. Dependence of the diffusion coefficient on the temperature. Given $v$ the coefficient $D_{\|}$is plotted vs $(c / T)$ for the Ohmic master equation and different coupling schemes: Caldeira-Leggett (X), sites (S), and bonds (B). The symbols are obtained numerically through an effective rate equation (see text). We also show results for the canonical ("Boltzmann") versions of the S/B master equation and for the semiclassical result in the case of $\mathrm{B}$ coupling. (The semiclassical result for $\mathrm{S}$ coupling is the same as $\mathrm{X}$ coupling). The naive expectation $D \propto\left\langle v^{2}\right\rangle$ is displayed for sake of comparison.

$v_{B}$, respectively. The dependence of $D$ on the temperature is plotted in Fig. 2. For sake of comparison we plot also the naive expectation $D \propto\left\langle v^{2}\right\rangle$, with $v=c \sin (p)$, where the average is over the canonical distribution. This naive expectation would be valid if the correlation time were independent of temperature (which is not the case). The high-temperature dependence is

$$
\left\langle v^{2}\right\rangle \approx\left[1+A\left(\frac{c}{T}\right)^{2}\right] \frac{c^{2}}{2}, \quad \text { with } A=-1 / 8 .
$$

In Sec. VII we obtain the same result also within the framework of an exact quantum treatment.

\section{B. S dissipation}

We shall explain that if the fluctuations of the bath are characterized by a finite correlation scale $\ell$, the semiclassical results for the transport coefficient are the same as for $\ell=\infty$. This rather trivial observation holds for any dispersion relation. Specifically for the tight-binding model Eq. (9) is $\ell$ independent. But this is not so in the quantum analysis. Here we analyze the other extreme limit of $\ell \sim a$, meaning that the bath fluctuations at different sites are uncorrelated. We obtain Eq. (10) with $A_{\|}=/ 16$ and $v \mapsto v_{S}$, which is not even the same sign when compared to the $\mathrm{X}$ dissipation result.

Technical note. For a tight-binding model the parameter $\eta$ is defined conventionally as for a two-site system (aka the spin-boson model). The definition via $-\eta \dot{x}$ is not practical because $x$ is a discrete variable. Still, in a semiclassical context, disregarding an ambiguity regarding the numerical prefactor, the dissipation parameter $\eta$ has the meaning of a friction coefficient, as for $\mathrm{X}$ coupling. With our standard conventions we get $C_{\|}=1 / 2$ (for $\mathrm{S}$ dissipation) instead of $C_{\|}=1$ (for $\mathrm{X}$ 
dissipation), which reflects a convention and not a profound difference. In contrast, the $A$ coefficients are independent of convention and reflect a quantum signature.

\section{B dissipation}

For completeness we also consider the case where the dissipation is due to uncorrelated noisy bonds (rather than sites). Here we have an additional term in the expression for the diffusion coefficient, namely, $D=D_{\|}+D_{\perp}$, where

$$
D_{\perp} \approx C_{\perp}\left[1+A_{\perp}\left(\frac{c}{T}\right)^{2}\right] v_{B}
$$

with $A_{\perp}=-1 / 4$. This additional term reflects extra spreading in the space due to stochastic hopping, as discussed in a previous publication [48]. The quantum fingerprints are not related to this term but to the $D_{\|}$term that arises due to the noise-induced spreading in $p$. For that term we find $A_{\|}=0$.

Technical note. The dissipation parameter $\eta_{B}$ is defined as for the spin-boson model. It is the "same" Ohmic bath as assumed for $\mathrm{S}$ dissipation, but the coupling term is different. We get $C_{\|}=1 / 6$ and $C_{\perp}=1$, as opposed to $\mathrm{X} / \mathrm{S}$ coupling for which $C_{\perp}=0$.

\section{Nonuniversality}

In general, for high temperatures the diffusion is composed of a term that originates from noncoherent hopping that is induced by the bath, namely, $D_{\perp}$ of Eq. (12), and from the interplay of noise with coherent hopping between sites, namely, $D_{\|}$of Eq. (10). The $\ell$ dependence of the latter is a quantum signature, and consequently, our result for $D_{\|}$reflects details of the dissipation mechanism.

The $A$ coefficients are not a matter of convention. Rather, they reflect the thermalization and the spreading mechanism, and hence indicate quantum manifestation. We summarize our results:

$$
A_{\|}= \begin{cases}-5 / 16 & \text { for } \mathrm{X} \text { coupling } \\ +1 / 16 & \text { for } \mathrm{S} \text { coupling } . \\ 0 & \text { for B coupling }\end{cases}
$$

Contrary to the X-coupling case, for local (S/B) dissipators the canonical $\rho$ is not the exact steady state and satisfies $\mathcal{L} \rho \sim$ $O\left(\beta^{3}\right)$ rather than zero. We shall explain that if we ad-hock correct the transition rates to get agreement with Boltzmann, the results for the $A$-s are modified as follows:

$$
A_{\|}= \begin{cases}-1 / 32 & \text { for S coupling } \\ -1 / 16 & \text { for B coupling }\end{cases}
$$

We emphasize again that the value of $A$ is a sensitive probe that is affected by the line shape of the spreading kernel. Therefore its precise value is nonuniversal but depends on the weights of the quantum transitions. For completeness we introduce in Sec. VIII results for intermediate values of $\ell$, demonstrating the crossover from $\mathrm{S}$ coupling $(\ell \sim a)$ to $\mathrm{X}$ coupling $(\ell \sim \infty)$.

\section{THE OHMIC DISSIPATOR}

The isolated chain is defined by the $\boldsymbol{H}^{(c)}$ Hamiltonian. The $\mathrm{X}$ dissipation scheme involves a single bath with inter- action term $-\boldsymbol{W F}$, where $\boldsymbol{W}$ is the position operator $\boldsymbol{x}$ and $F$ is a bath operator that induces Ohmic fluctuations with intensity $v$. More generally, we assume a disordered thermal environment that is composed of numerous uncorrelated baths such that the interaction term is $\sum_{\alpha} \boldsymbol{W}_{\alpha} F_{\alpha}$, where $\alpha$ labels different locations. For $\mathrm{S}$ dissipation $\boldsymbol{W}_{\alpha}=\left|x_{\alpha}\right\rangle\left\langle x_{\alpha}\right|$, leading to a fluctuating potential that dephases the different sites. For B-dissipation $\boldsymbol{W}_{\alpha}=\left|x_{\alpha}+1\right\rangle\left\langle x_{\alpha}\right|+$ H.c., which induces incoherent hopping between neighboring sites. The Ohmic dissipator $\mathcal{L}^{(\mathrm{X} / \mathrm{S} / \mathrm{B})} \rho$ takes the form $[48,49]$

$$
-\sum_{\alpha}\left(\frac{v}{2}\left[\boldsymbol{W}_{\alpha},\left[\boldsymbol{W}_{\alpha}, \rho\right]\right]+\frac{\eta}{2} i\left[\boldsymbol{W}_{\alpha},\left\{\boldsymbol{V}_{\alpha}, \rho\right\}\right]\right),
$$

where $\eta=v /(2 T)$ is the friction coefficient, and

$$
\boldsymbol{V}_{\alpha} \equiv i\left[\boldsymbol{H}^{(c)}, \boldsymbol{W}_{\alpha}\right] \text {. }
$$

The friction terms represent the response of the bath to the rate of change of the $\boldsymbol{W}_{\alpha}$. For $\mathrm{X}$ dissipation $\boldsymbol{V}=c \sin (\boldsymbol{p})$ is the velocity operator. If we treat the friction term of Eq. (15) in a semiclassical way, the expression for the dissipator in the Wigner phase-space representation $\rho_{w}(R, P)$ takes the familiar Fokker-Plank (FP) form with $v=c \sin (P)$, namely,

$$
\mathcal{L}^{\mathrm{FP}} \rho_{w}=\frac{v}{2} \partial_{P}^{2}\left[\rho_{w}\right]-\partial_{P}\left[\left(f_{0}-\eta v\right) \rho_{w}\right],
$$

which is a sum of momentum-diffusion and momentum-drift terms. For the sake of later reference we have added to the friction force $(-\eta v)$ a constant field $f_{0}$.

The $\mathrm{X}$ dissipator leads to canonical steady state for any friction and for any temperature. This is not the case for $\mathrm{S} / \mathrm{B}$ dissipation, for which the agreement of the steady state with the canonical result is guaranteed only to second order in $\eta$. The reason for that is related to the proper identification of the "small parameter" that controls the deviation from canonical thermalization. The $\mathrm{X}$ dissipator induces transitions between neighboring momenta, and therefore the small parameter is $\Delta / T$, where the level spacing $\Delta$ goes to zero in the $L \rightarrow \infty$ limit, where $L$ is the length of the chain. But for local baths, the coupling is to local scatterers that create transitions to all the levels within the band. Therefore the small parameter is $c / T$, and canonical thermalization is expected only for $c / T<1$.

\section{SEMICLASSICAL ANALYSIS FOR X DISSIPATION}

We shall argue later that for $\mathrm{X}$ dissipation the semiclassical dynamics that is generated by $\mathcal{L}^{\mathrm{FP}}$ is exact for the purpose of the $A$ coefficient evaluation. Here we present the semiclassical solution.

In Sec. IV A below we find the steady-state momentum distribution in the presence of a constant field $f_{0}$. In Sec. IV B we obtain for the weak field $\langle v\rangle=\mu f_{0}$, where $\mu$ is the mobility. Then the diffusion coefficient is deduced from the Einstein relation, namely, $D=\mu T$, leading to Eq. (9).

Optionally, we can calculate directly the velocity-velocity correlation function $\langle v(t) v(0)\rangle$ in the absence of an external field. This requires a rather complicated recursive procedure, see Appendix A. The diffusion coefficient is obtained via

$$
D=\int_{0}^{\infty} d t\langle v(t) v(0)\rangle \text {. }
$$


The same result is obtained, namely, Eq. (9). Later we shall calculate the diffusion in a proper quantum calculation, and this again yields the same result; see Sec. VII.

\section{A. The steady state}

We consider a Brownian particle that is described by Eq. (6) under the influence of a thermal nondisordered fluctuating field (X coupling). Below we set $a=1$ for the lattice constant. A fully quantum treatment of this model has been introduced by [7,21], with focus on the low-temperature QBM regime, while here we focus on the high-temperature regime. The semiclassical equations of motion are formally obtained by the substitution $f_{0} \mapsto F(t)$, where the total force $F(t)=f_{0}+f(t)-\eta \dot{x}$ includes a stochastic term that has zero average with correlation function $\left\langle f(t) f\left(t^{\prime}\right)\right\rangle=v \delta(t-$ $\left.t^{\prime}\right)$ and an associated friction term with coefficient $\eta$, in additional to the bias term $f_{0}$. Thus we get the Langevin equation

$$
\begin{gathered}
\dot{x}=\frac{\partial H}{\partial p}=c \sin (p), \\
\dot{p}=-\frac{\partial H}{\partial x}=f_{0}-\eta \dot{x}+f(t) .
\end{gathered}
$$

The steady state for $p$ is solved by inserting Eq. (19) to Eq. (20). Changing notation $p \mapsto \varphi$, and $u(\varphi)=f_{0}-\eta c \sin (\varphi)$, and $D_{\varphi}=(1 / 2) v$, one gets the equation $\dot{\varphi}=u(\varphi)+f(t)$, with the associated Fokker-Planck equation

$$
\frac{\partial}{\partial t} \rho(\varphi, t)=-\frac{\partial}{\partial \varphi} I
$$

with

$$
\begin{aligned}
I & =u(\varphi) \rho-D_{\varphi} \frac{\partial \rho}{\partial \varphi} \equiv-D_{\varphi}\left[V^{\prime}(\varphi) \rho+\frac{\partial \rho}{\partial \varphi}\right] \\
& =-D_{\varphi} e^{-V(\varphi)} \frac{\partial}{\partial \varphi}\left[e^{V(\varphi)} \rho\right] .
\end{aligned}
$$

This equation describes motion in a tilted potential

$$
\begin{aligned}
V(\varphi) & =-\frac{\eta c}{D_{\varphi}} \cos (\varphi)-\frac{f_{0}}{D_{\varphi}} \varphi \\
& \equiv W(\varphi)-\mathcal{E} \varphi .
\end{aligned}
$$

The nonequilibrium steady-state (NESS) solution is

$$
\rho(\varphi)=\left[C-I \int_{0}^{\varphi} \frac{e^{V\left(\varphi^{\prime}\right)}}{D_{\varphi}} d \varphi^{\prime}\right] e^{-V(\varphi)},
$$

where the integration constant $C$ is determined by the periodic boundary conditions $\rho_{0}(0)=\rho_{0}(2 \pi)$, namely,

$$
C=\frac{I}{1-e^{-2 \pi \mathcal{E}}} \int_{0}^{2 \pi} \frac{e^{V\left(\varphi^{\prime}\right)}}{D_{\varphi}} d \varphi^{\prime} .
$$

Simplifying, the final expression for the NESS is

$$
\rho(\varphi)=\frac{I}{1-e^{-2 \pi \mathcal{E}}}\left[\int_{0}^{2 \pi} \frac{d r}{D_{\varphi}} e^{W(\varphi+r)-\mathcal{E} r}\right] e^{-W(\varphi)},
$$

where the $\varphi$ current $I$ is determined by normalization.

\section{B. The transport coefficients}

Reverting to the original notations the first-order result in $f_{0}$ is $I=\left[2 \pi \mathrm{I}_{0}^{2}(c / T)\right]^{-1} f_{0}$, where $\mathrm{I}_{n}(x)$ is the modified Bessel function. For zero field the canonical distribution is recovered:

$$
\rho(p) \propto \exp [-W(p)]=\exp [(c / T) \cos (p)] .
$$

Averaging over Eq. (20) and using $\langle\dot{p}\rangle=2 \pi I$, one obtains

$$
\langle\dot{x}\rangle=[1-2 \pi I] \frac{f_{0}}{\eta}=\left[1-\mathrm{I}_{0}^{-2}\left(\frac{c}{T}\right)\right] \frac{f_{0}}{\eta} \equiv \mu f_{0},
$$

where $\mu$ is the so-called linear mobility. This result for $\mu$ is consistent with direct calculation of $D$ in accordance with the Einstein relation, namely, $\mu=D / T$. The direct calculation of $D$ is more involved. It is obtained by calculating the variance of $x$, after time $t$, for a particle initially located at $x=0$ :

$$
\left\langle x^{2}\right\rangle=c^{2} \int_{0}^{t} \int_{0}^{t} d t^{\prime} d t^{\prime \prime}\left\langle\sin \left(\varphi_{t^{\prime}}\right) \sin \left(\varphi_{t^{\prime \prime}}\right)\right\rangle \equiv 2 D t .
$$

Defining $S_{1}$ as the area of the sine correlation function we write $D=c^{2} S_{1}$. The calculation of $S_{1}$ is outlined in Appendix A.

\section{SEMICLASSICAL ANALYSIS FOR S DISSIPATION}

In the semiclassical treatment $x$ is regarded as as continuous coordinate and therefore we write

$$
\boldsymbol{W}_{\alpha}=u_{\alpha}(\boldsymbol{x})=u\left(\boldsymbol{x}-x_{\alpha}\right),
$$

which involves a short-range interaction potential $u(r)$. The fluctuating potential is

$$
\mathcal{U}(x, t)=\sum_{\alpha} F_{\alpha}(t) u\left(x-x_{\alpha}\right) .
$$

In the semiclassical analysis we define $v$ as the variance of $f=-\mathcal{U}^{\prime}(x, t)$. These fluctuations have the same intensity at any $x$ because we assume that the $x_{\alpha}$ are homogeneously distributed. It follows automatically that $\eta=v / 2 T$ is the friction coefficient, as in the case of X dissipation. See [40] for details. So in the semiclassical description we get the same Langevin equation, irrespective of the correlation distance $\ell$ that is determined by the width of $u(r)$.

In the tight-binding quantum model, we define $v_{S}$ as the variance of the on-site fluctuation of the potential. With that we associate a fluctuating force intensity

$$
v=\frac{1}{\ell^{2}} v_{S},
$$

where $\ell$ is the correlation scale. We set $\ell \sim a$, where $a=1$ is the lattice constant. Consequently $v$, up to numerical factor, is the same as $v_{S}$. The price for having a vague definition for $v$ is the prefactor $C$ that we get in the formula for $D$. This prefactor reflects that the semiclassical limit has an inherent numerical ambiguity due to the residual freedom in the choice of $u(r)$.

\section{SEMICLASSICAL ANALYSIS FOR B DISSIPATION}

Using the same prescription as for the S-dissipation case, and ignoring commutation issues, we write 
$\sum_{\alpha}\left(\left|x_{\alpha}+1\right\rangle\left\langle x_{\alpha}\right|+\right.$ H.c. $)$ as $[2 \cos (\boldsymbol{p})]|\boldsymbol{x}\rangle\langle\boldsymbol{x}|$, and get for the B-coupling term

$$
\boldsymbol{W}_{\alpha}=[2 \cos (\boldsymbol{p})] u_{\alpha}(\boldsymbol{x}) .
$$

This means that motion with momentum $|p| \sim \pi / 2$ is not affected by the baths. This is an artifact of the semiclassical treatment and does not hold for the quantum dynamics. Still, the semiclassical perspective provides some insight that helps to clarify how Eq. (12) comes out.

The equations of motion that are derived from the full Hamiltonian are of Langevin type:

$$
\begin{gathered}
\dot{x}=\left[c+2 \sum_{\alpha} u_{\alpha}(x) F_{\alpha}(t)\right] \sin (p), \\
\dot{p}=\left[2 \sum_{\alpha} u_{\alpha}^{\prime}(x) F_{\alpha}(t)\right] \cos (p) .
\end{gathered}
$$

For infinite temperature the $F_{\alpha}$ are uncorrelated white noise terms, with some intensity proportional to $v_{B}$. Therefore we get from Eq. (33) diffusion in $p$ with coefficient $v_{p}=(1 / \ell)^{2}[2 \cos (a p)]^{2} v_{B}$ and from Eq. (32) extra diffusion in $x$ with coefficient $v_{x}=(a)^{2}[2 \cos (a p)]^{2} v_{B}$, where $\ell \approx a$ and $a=1$. The latter term, after momentum averaging, is responsible for getting the $D_{\perp}$ term in Eq. (12). For a particle that moves with constant momentum $p$, ignoring the variation in $p$, the velocity-velocity correlation decays as $\exp \left(-v_{x} t\right)$ due to this $x$ diffusion. This leads to an extra Drude term $D_{\|}=v^{2} / v_{x}$ that diverges at $p=\pi / 2$. However, taking the variation of the momentum into account, this divergence has zero measure and the final result is finite, leading to the first term in Eq. (10) with $C_{\|}=0.49$. For finite temperature the fluctuations gain a nonzero average $\left\langle F_{\alpha}\right\rangle=2 \eta_{B}\left(\left[u_{\alpha}(x) \sin (p)\right] \dot{p}-\left[u_{\alpha}^{\prime}(x) \cos (p)\right] \dot{x}\right)$, where $\eta_{B}=v_{B} / T$, leading to canonical-like thermalization and overestimated $A_{\|}=-0.2$. The results for $A_{\|}$and $C_{\|}$were obtained using a procedure that is described in Sec. VIII, where we treat the quantum and the semiclassical on equal footing; the latter can be regarded as a special case of the former.

\section{THE QUANTUM ANALYSIS}

The quantum evolution is generated by $\mathcal{L}$ of Eq. (7) with the dissipators of Eq. (15), and it can be written as sum of Hamiltonian, noise, and friction terms, namely, $\mathcal{L}=c \mathcal{L}^{(c)}+v \mathcal{L}^{(v)}+\eta c \mathcal{L}^{(\eta)}$. Various representations can be used, notably the Wigner and the Bloch representations see Appendix B. For the purpose of finding the spectrum (and from that the transport coefficients), it is most convenient to use the latter (Bloch), as explained below.

The elements of the supervector $\rho$ are given in the standard representation by $\rho(R, r) \equiv\langle R+r / 2|\rho| R-r / 2\rangle$, and in Dirac notation we write $\rho=\sum_{R, r} \rho(R, r)|R, r\rangle$. The supermatrix $\mathcal{L}$ is invariant under $R$ translations, and therefore it is convenient to switch to a Bloch representation $\rho(q ; r)$ where $\mathcal{L}$ decomposes into $q$ blocks. In the $q$ subspace we have the following expressions (Appendix C):

$$
\begin{aligned}
\mathcal{L}^{(c)} & =+\sin (q / 2)\left(\mathcal{D}_{\perp}-\mathcal{D}_{\perp}^{\dagger}\right) \\
\mathcal{L}^{\left(v_{X}\right)} & =-(1 / 2) \hat{r}^{2}
\end{aligned}
$$

$$
\begin{aligned}
\mathcal{L}^{\left(\eta_{X}\right)}= & \cos (q / 2) \frac{\hat{r}}{2}\left(D_{\perp}-D_{\perp}^{\dagger}\right) \\
\mathcal{L}^{\left(v_{S}\right)}= & -1+1|0\rangle\langle 0| \\
\mathcal{L}^{\left(\eta_{S}\right)}= & \frac{\cos (q / 2)}{2}\left(\mathcal{D}_{\perp}+D_{\perp}^{\dagger}+| \pm 1\rangle\langle 0|-| 0\rangle\langle \pm 1|\right) \\
\mathcal{L}^{\left(v_{B}\right)}= & -2+2 \cos (q)|0\rangle\langle 0|+(|1\rangle\langle-1|+|-1\rangle\langle 1|) \\
\mathcal{L}^{\left(\eta_{B}\right)}= & \frac{1}{2} \cos (q / 2)\left(\mathcal{D}_{\perp}+\mathcal{D}_{\perp}^{\dagger}\right) \\
& +\frac{1}{2} \cos (3 q / 2)(| \pm 1\rangle\langle 0|-| 0\rangle\langle \pm 1|) \\
& +\frac{1}{2} \cos (q / 2)(|\mp 2\rangle\langle \pm 1|-| \pm 1\rangle\langle\mp 2|) .
\end{aligned}
$$

The subscripts $\mathrm{X} / \mathrm{S} / \mathrm{B}$ distinguish the different coupling schemes, and $\mathcal{D}_{\perp}=|r+1\rangle\langle r|$ is the displacement operator in $r$ space.

\section{A. Extracting the diffusion coefficient}

To obtain the diffusion coefficient, we consider the spectrum of $\mathcal{L}$ for a finite system of $L$ sites. In the Bloch representation the equation $\mathcal{L} \rho=-\lambda \rho$ decomposes into $q$ blocks. For a given $q$ we have a tight-binding equation in the $|r\rangle$ basis. For example, $\mathcal{L}^{(c)}$ induces near-neighbor hopping in $r$. The eigenvalues for a given $q$ are labeled $\lambda_{q, s}$, where $s$ is a band index. The long-time dynamics is determined by the slow $(s=0)$ modes. Specifically, the diffusion coefficient is determined by the small $q$ expansion:

$$
\lambda_{q, 0}=D q^{2}+\mathcal{O}\left(q^{4}\right) .
$$

The NESS eigenvector belongs to the $q=0$ block, and for $\eta=0$ it is given by $|r=0\rangle$. Nonzero $q$ and $\eta$ can be treated as a perturbation. The key observation is that in order to get an exact result for $D$, it is enough to use second-order perturbation theory in $q$. The outcome of this procedure is the analytical expression for $D$ with the associated results for the $A$ coefficients. Extra technical details are provided in the next section.

\section{B. Perturbation theory}

We use perturbation theory to find the eigenvalue $\lambda_{q, 0}$ of $\mathcal{L}^{(q)}$, from which we can obtain $D$. We regard the Bloch quasimomentum $q$ and the friction $\eta$ as the perturbation. For $q=\eta=0$ the state $|r=0\rangle$ is an exact eigenstate that is associated with the eigenvalue $\lambda=0$. Due to the perturbation it is mixed with neighboring $|r\rangle$ states. We outline below how we get analytical expressions for $\lambda_{q, 0}$ to any order in $q$ and $\eta$. In practice, we go up to second order.

In the following we demonstrate how we perform perturbation theory for the X-coupling scheme. The same method is used for the S/B coupling schemes, either with the Ohmic dissipators or with the Boltzmann dissipators. We would like to diagonalize the $q$ block

$$
\begin{aligned}
\mathcal{L}^{(q)}= & c \mathcal{L}^{(c)}+v \mathcal{L}^{\left(v_{X}\right)}+(c \eta) \mathcal{L}^{\left(\eta_{X}\right)} \\
= & c \sin (q / 2)\left(\mathcal{D}_{\perp}-\mathcal{D}_{\perp}^{\dagger}\right)-(v / 2) \hat{r}^{2} \\
& +(c \eta) \cos (q / 2) \frac{\hat{r}}{2}\left(D_{\perp}-D_{\perp}^{\dagger}\right) .
\end{aligned}
$$


Each such block produces eigenvalues $\mathcal{L}^{(q)}|s\rangle=-\lambda_{q, s}|s\rangle$, that are distinguished by the index $s$. We are interested in the slowest mode $\lambda_{q, 0}$. The NESS is the eigenvector that corresponds to the zero eigenvalue. It belongs to the $q=0$ block, which results from probability conservation. In the Bloch representation, probability conservation means that $\langle 0| \mathcal{L}^{(0)}=0$. To obtain the eigenvalues to order $q^{2}$, it is enough to Taylor expand the operator to that order. Accordingly,

$$
\begin{aligned}
\mathcal{L}^{(q)}= & -(v / 2) \hat{r}^{2}+c(q / 2)\left(\mathcal{D}_{\perp}-\mathcal{D}_{\perp}^{\dagger}\right) \\
& +(c \eta)\left[1-(q / 2)^{2}\right] \frac{\hat{r}}{2}\left(D_{\perp}-D_{\perp}^{\dagger}\right) .
\end{aligned}
$$

The first term is the zero-order term. Here (for X coupling) it is diagonal in $r$. For the other coupling schemes it is not necessarily diagonal in $r$, but for any of them $|r=0\rangle$ is an eigenstate of the zero-order term.

To find the eigenvalue $\lambda_{q, 0}$ via perturbation theory one has to sum over different paths that begin and end in $r=0$. In the case of Eq. (37) these paths are composed of hops between near-neighbor sites. Second-order contributions involve terms with $\left\langle 0\left|\mathcal{L}^{(q)}\right| r\right\rangle\left\langle r\left|\mathcal{L}^{(q)}\right| 0\right\rangle$, with $r \neq 0$. Each transition involves a factor $c q$ or $(c \eta)$, or $\left(c \eta q^{2}\right)$. Hence only the sites $|r| \leqslant 2$ contribute to the perturbed eigenvalue up to order $\eta^{2} q^{2}$. Furthermore, the $\left(c \eta q^{2}\right)$ transitions are always multiplied by other $\mathcal{O}(q)$ transitions and therefore can be ignored in any secondorder expansion.

From the above it should be clear that for $\mathrm{X}$ coupling the matrix that should be diagonalized is

$$
\mathcal{L}^{(q)} \mapsto \frac{1}{2}\left(\begin{array}{ccccc}
-4 v & 2 c \eta-c q & 0 & 0 & 0 \\
-c \eta+c q & -v & c \eta-c q & 0 & 0 \\
0 & c q & 0 & -c q & 0 \\
0 & 0 & c \eta+c q & -v & -c \eta-c q \\
0 & 0 & 0 & 2 c \eta+c q & -4 v
\end{array}\right) .
$$

A convenient way to obtain the analytical result is to write the characteristic equation $\operatorname{det}\left[\lambda+\mathcal{L}^{(q)}\right]=0$ with the above (truncated) matrix and to substitute an expansion $\lambda_{q, 0}=\sum_{n} a_{n} q^{n}$. Then we solve for the coefficients $a_{n}$ iteratively. The outcome is expanded in $\eta$ to order $\eta^{2}$. Note that to go beyond second order in $\eta$ does not makes sense, because the Ohmic master equation and the associated NESS are valid only up to this order.

\section{EFFECTIVE STOCHASTIC DESCRIPTION}

The propagation of the Wigner distribution function $\rho_{w}(R, P)$ is generated by a kernel $\mathcal{L}\left(R, P \mid R_{0}, P_{0}\right)$ that is obtained from Eq. (34) in a straightforward manner via the Fourier transform, Appendix B. For simulations of the longtime spreading it is enough to approximate $\mathcal{L}$ in a way that is consistent with second-order perturbation theory in $q$. As explained in the previous paragraph, such an approximation provides an exact result as far as $D$ calculation is concerned. Replacing $\sin (q / 2)$ by $(q / 2)$, the $\mathcal{L}^{(c)}$ term by itself generates classical motion in the $X$ direction with velocity $v=c \sin (P)$. In the quantum calculation this motion is decorated by a Bessel function but $D$ is not affected. The $\cos (q)$ in the $\mathcal{L}^{\left(v_{B}\right)}$, after expansion to second order and Fourier transform, leads to an $x$ diffusion term that is responsible for the $C_{\perp}$ contribution in Eq. (12). As far as this term is concerned, there is no difference between the quantum and the semiclassical picture, and therefore we ignore it in the subsequent analysis. The cosine factors in the other dissipators can be replaced by unity. The reason is as follows: by themselves those cosine terms do not lead to any diffusion; only when combined with the $\mathcal{L}^{(c)}$ term do they lead to the Drude-type $C_{\|}$contribution in Eq. (10); the $\mathcal{L}^{(c)}$ is already first order in $q$; and hence there is no need to expand the cosines beyond zero order.

\section{A. The effective rate equation}

With the approximations that were discussed in the previous paragraph (excluding for presentation purpose the trivial $R$ diffusion in the case of B-dissipation), we find that the evolution of the Wigner function is generated by a stochasticlike kernel $\mathcal{L}\left(R, P \mid R_{0}, P_{0}\right)=\mathcal{W}\left(P \mid P_{0}\right) \delta\left(R-R_{0}\right)$. The explicit expressions for infinite temperature $(\eta=0)$ are

$$
\begin{gathered}
\mathcal{W}^{\left(v_{X}\right)}\left(P \mid P_{0}\right)=\left(\frac{L}{2 \pi}\right)^{2} \frac{v}{2} \delta_{P, P_{0} \pm(2 \pi / L)}, \\
\mathcal{W}^{\left(v_{S}\right)}\left(P \mid P_{0}\right)=\left(\frac{\nu_{S}}{L}\right), \\
\mathcal{W}^{\left(v_{B}\right)}\left(P \mid P_{0}\right)=\left(\frac{v_{B}}{L}\right) 4 \cos ^{2}\left(\frac{P+P_{0}}{2}\right) .
\end{gathered}
$$

These are the transition rates $\left(P \neq P_{0}\right)$, while the diagonal elements of $\mathcal{W}$ are implied by conservation of probability. For $\mathrm{X}$ dissipation Eq. (38) describes local spreading of momentum, which is in complete correspondence with the semiclassical analysis. The noise intensity is reflected in the second moment:

$$
v=\sum_{p} W(p) p^{2},
$$

where $p=\left(P-P_{0}\right)$. This implies consistency with the Langevin equation, Eq. (1). Optionally, Eq. (38) can be regarded as the discrete version of the Fokker-Plank equation, Eq. (17). For S dissipation Eq. (39) describes quantum diffractive spreading. In the latter case, if the dynamics were treated semiclassically one would obtain the same result as for $\mathrm{X}$ dissipation, namely, Eq. (38), with prefactor of order unity that can be by rescaled to unity by adopting the appropriate convention for the definition of $v$. In other words, the coupling strength to the bath should be redefined such that $v$ is the second moment of $\mathcal{W}\left(P \mid P_{0}\right)$ irrespective of the line shape. Similarly, if the dynamics were treated semiclassically for the B coupling, one would obtain Eq. (38) multiplied by $4 \cos ^{2}(P)$, as implied by the semiclassical analysis.

The result for $\mathcal{W}$ for finite temperature, in leading order in $\eta$ (which serves here as a dimensionless version of the inverse temperature) can be written as

$$
\mathcal{W}\left(P \mid P_{0}\right)=\mathcal{W}^{(v)}\left(P \mid P_{0}\right) \exp \left[-\frac{E(P)-E\left(P_{0}\right)}{2 T}\right],
$$

where $E(P)=-c \cos (P)$. More precisely, if we incorporate the $\mathcal{L}^{(\eta)}$ term of the Ohmic master equation, we get Eq. (42) with $e^{x} \mapsto(1+x)$. This reflects the well-known observation that the Ohmic approximation satisfies detailed balance to second order in $\eta$. Accordingly, the Ohmic steady state agrees to second order with the canonical steady state, $\rho_{\mathrm{SS}}(P) \propto \exp [-E(P) / T]$. 


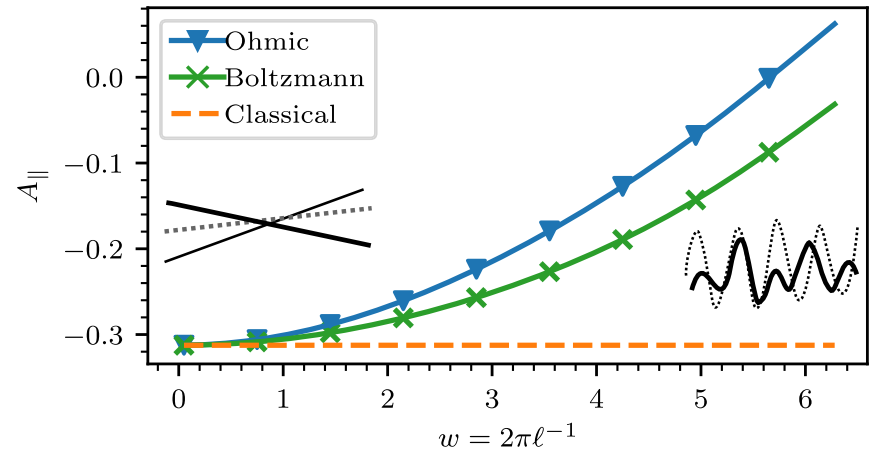

FIG. 3. Quantum nonuniversality. The dependence of the coefficient $A_{\|}$on $\ell$. The insets caricature the fluctuating potential for large (left) and for small (right) values of $\ell$. In the semiclassical analysis the result (dashed orange line) is universal, independent of $\ell$. In the quantum analysis we obtain an interpolation between the $\mathrm{X}$-coupling and the S-coupling case Eq. (13). We plot results (see text) for the Ohmic (blue triangles) and for the Boltzmann-corrected (green crosses) versions of the master equation.

\section{B. Analytical and numerical estimates}

The stochastic description allows a convenient way to obtain exact results for $D$ either analytically or numerically. Analytically, we use the same procedure as in the quantum case, namely, given the dissipator $\mathcal{L}^{(q)}$, we extract $D$ from Eq. (35). The relation between $\mathcal{L}^{(q)}$ and the stochastic kernel is

$$
\begin{aligned}
\left\langle r\left|\mathcal{L}^{(q)}\right| r_{0}\right\rangle & =\left\langle r, q|\mathcal{L}| r_{0}, q\right\rangle \\
& =\frac{1}{L} \sum_{P, P_{0}} \mathcal{W}\left(P \mid P_{0}\right) e^{i P r-i P_{0} r_{0}} .
\end{aligned}
$$

The X-coupling and $\mathrm{S}$-coupling schemes provide two extremes, with $\ell=L$ and $\ell=1$, respectively. This is mirrored in the infinite-temperature kernel $\mathcal{W}$ of Eqs. (38) and (39). On equal footing we can interpolate between the two extremes by introducing a kernel of width $2 \pi / \ell$. Then we use Eq. (42) to get the finite-temperature kernel. The calculation of $\mathcal{L}^{(q)}$ using Eq. (43) is provided in Appendix D. The result for $A_{\|}$ is displayed in Fig. 3. Note that the convention regarding the prefactor in $\mathcal{W}^{(v)}$ plays no role in the determination of $A_{\|}$.

At this point we have to emphasize again that for the "Ohmic" results we use the prescription $e^{x} \mapsto(1+x)$, as explained after Eq. (42). If we perform the calculation literally using Eq. (42) we get Eq. (14) instead of Eq. (13). Note that the same results are obtained with $e^{x} \mapsto\left(1+x+(1 / 2) x^{2}\right)$, because higher orders do not affect the expansion in Eq. (35). The difference between Eqs. (14) and (13) reflects the limited accuracy of the Ohmic master equation with respect to the small parameter $c / T$.

The analytical results for the $A_{\|}$coefficients that are plotted in Fig. 3 are derived and displayed in Appendix D. Here we write expressions that approximate very well the exact results:

$$
\begin{gathered}
A_{\|} \approx-\frac{5}{16}\left[1-\frac{6}{5}\left(\frac{a}{\ell}\right)^{2}\right] \quad[\text { Ohmic] } \\
A_{\|} \approx-\frac{5}{16}\left[1-\frac{9}{10}\left(\frac{a}{\ell}\right)^{2}\right] \quad \text { [Boltzmann]. }
\end{gathered}
$$

Note that this practical approximation provides the exact results for both $\mathrm{X}$ coupling $(\ell=\infty)$ and $\mathrm{S}$ coupling $(\ell=a=1)$.

In Fig. 2 we test the analytical approximation Eq. (10) against exact numerical calculation that is based on the effective rate equation. In the numerical procedure the diffusion coefficient $D$ is calculated using Eq. (18). The momentum spreading kernel is $K(t) \equiv \exp (\mathcal{W} t)$, and the velocity is $v_{P}=c \sin (P)$. Accordingly,

$$
\langle v(t) v(0)\rangle=\sum_{P, P_{0}} v_{P}\left[K_{P, P_{0}}(t)\right] v_{P_{0}} \rho_{\mathrm{SS}}\left(P_{0}\right) .
$$

If we perform the calculation literally using Eq. (42) we get results that agree with Eq. (14). If, on the other hand, we use for $\mathcal{W}$ the Ohmic expression [as specified after Eq. (42)] we get results that agree with Eq. (13). Note that for $\rho_{S S}$ we can use the canonical steady state, because it agrees with the Ohmic steady state to second order.

\section{DISCUSSION}

The prototype Caldeira-Leggett model corresponds to the standard Langevin equation where the dispersion relation is $v=(1 / \mathrm{m}) p$. In the tight-binding framework we have the identification $\mathrm{m} \mapsto 1 /\left(c a^{2}\right)$, where $a$ is the lattice constant. There is a crossover to standard QBM as $\theta \equiv T / c$ is lowered. It is illuminating to summarize this crossover in terms of mobility. Using the Einstein relation Eqs. (10) and (12) imply

$$
\mu=\frac{D}{T}=\frac{B(\theta)}{\eta}+2 Q(\theta) \eta,
$$

where the $B(\theta)$ term is related to the coherent hopping, and the $Q(\theta)$ term is due to bath-induced incoherent hopping. We believe that this functional form is rather robust and apply it to any type of dissipation mechanism. The traditional result is the first term with $B(\theta)=1$, while Eq. (10) implies that for large $\theta$ the result is

$$
B(\theta) \propto(1 / \theta)^{2}+A_{\|}(1 / \theta)^{4} .
$$

We have shown how $A_{\|}$depend on $\ell$, with emphasis on the extreme limits of $\mathrm{X}$ coupling and $\mathrm{S}$ coupling. We conclude that the $A$ coefficients provide a way to probe the underlying mechanism of dissipation and to identify the high-temperature fingerprints of quantum mechanics.

It should be instructive to demonstrate experimentally that $\mu(T ; \ell)$ indeed depends on $\ell$. Reference [50] provides an experimental demonstration of measuring mobility versus temperature for a semiconductor device, while Ref. [51] reviews experimental methods used to extract the mobility in organic semiconductors. Consider the possibility of fabricating a metallic gate that produces thermal electrostatic fluctuations. Metals that differ in their granularity are characterized by a different form factor, with different correlation scale $\ell$. Thus it would be possible to demonstrate that $\ell$ has significance. Hopefully, it would be possible to further extract, experimentally, the nonuniversal dependence of $A_{\|}$on the correlation distance $\ell$ and to test the prediction of Fig. 3. 


\section{ACKNOWLEDGMENTS}

This research was supported by the Israel Science Foundation (Grant No. 283/18). We thank Muntaser Naamneh for his advice on the experimental aspect.

\section{APPENDIX A: THE SINE CORRELATION FUNCTION}

First we recall that for zero field the steady state is an equilibrium canonical state $\rho(\varphi) \propto \exp [-W(\varphi)]$, where $W(\varphi)=z \cos (\varphi)$, and $z=(c / T)$. At equilibrium we have

$$
w_{n} \equiv\langle\cos (n \varphi)\rangle=\frac{\mathrm{I}_{n}(z)}{\mathrm{I}_{0}(z)}
$$

We define $S_{n}$ as the area of the sine-sine correlation function $s_{n}(t)=\left\langle\sin \left(n \varphi_{t}\right) \sin \left(\varphi_{0}\right)\right\rangle$, namely,

$$
S_{n}=\int_{0}^{\infty} s_{n}(t) d t, \quad n=0,1,2, \ldots
$$

Eventually we are interested only in $S_{1}$, but for the derivation we define a full set of sine correlation functions. Explicitly these are written as

$$
\begin{aligned}
s_{n}(t) & =\int_{0}^{2 \pi}\left\langle\sin \left(n \varphi_{t}\right)\right\rangle_{0} \sin \left(\varphi_{0}\right) \rho\left(\varphi_{0}\right) d \varphi_{0} \\
& =\int_{0}^{2 \pi}\langle\sin (n \varphi)\rangle_{t} \sin \left(\varphi_{0}\right) \rho\left(\varphi_{0}\right) d \varphi_{0} .
\end{aligned}
$$

The average without a subscript assumes an equilibrium state, while the average with subscript " 0 " indicates an initial condition $\varphi_{0}$ and assumes a Langevin picture. The subscript " $t$ " indicates an expectation value after time $t$ within the framework of the associated Fokker-Planck picture. Initially we have

$$
\begin{aligned}
s_{n}(0) & =\langle\sin (n \varphi) \sin (\varphi)\rangle=\frac{1}{2} \frac{\mathrm{I}_{n-1}(z)-\mathrm{I}_{n+1}(z)}{\mathrm{I}_{0}(z)} \\
& =\frac{n}{z} \frac{\mathrm{I}_{n}(z)}{\mathrm{I}_{0}(z)} .
\end{aligned}
$$

In order to find $s_{n}(t)$ at later times, we realize that it satisfies the same equation of motion as that of $\left\langle\sin \left(n \varphi_{t}\right)\right\rangle_{0}$, where 0 indicates any initial state. This is known as the "regression theorem." The adjoint equation for any observable $A(\varphi)$ is

$$
\frac{\partial}{\partial t}\langle A(\varphi)\rangle_{t}=\left\langle D_{\varphi}\left(\frac{\partial^{2}}{\partial \varphi^{2}}-W^{\prime}(\varphi) \frac{\partial}{\partial \varphi}\right) A(\varphi)\right\rangle_{t}
$$

Substituting $A(\varphi):=\sin (\varphi) \sin \left(\varphi_{0}\right)$ and integrating over time, one obtains a recursive equation for the $S_{n}$ :

$$
s_{n}(0)=n^{2} \frac{v}{2} S_{n}+n \frac{\eta c}{2}\left(S_{n+1}-S_{n-1}\right),
$$

with the boundary conditions $S_{0}=S_{\infty}=0$. At this point it is useful to realize that from Eq. (A5) with $A(\varphi):=\cos (n \varphi)$ it follows that the stationary values $w_{n}$ of Eq. (A1) obey Eq. (A6) with zero on the left-hand side. It is therefore useful to substitute $S_{n}:=w_{n} \tilde{S}_{n}$ in order to get a first-order difference equation for $\tilde{S}_{n}$ that can be solved by recursion. The procedure is explained with details in Sec. VII of Ref. [52] and leads to the solution

$$
\begin{aligned}
S_{1} & =-\frac{1}{\eta c} \sum_{n=1}^{\infty} \frac{(-1)^{n}}{n} s_{n}(0) w_{n}=-\frac{v}{(\eta c)^{2}} \sum_{n=1}^{\infty}(-1)^{n}\left[\frac{\mathrm{I}_{n}(z)}{\mathrm{I}_{0}(z)}\right]^{2} \\
& =\frac{v}{2(\eta c)^{2}}\left[1-\mathrm{I}_{0}^{-2}(z)\right],
\end{aligned}
$$

where we used the completeness relation

$$
1=\mathrm{I}_{0}^{2}(z)+2 \sum_{n} \mathrm{I}_{n}^{2}(z)(-1)^{n} .
$$

\section{APPENDIX B: THE WIGNER PHASE-SPACE REPRESENTATION}

Here we treat $(x, p)$ as extended continuous coordinates and derive the standard Wigner representation for the quantum propagation in the absence of dissipators. The elements of $\rho$ are given in the standard space representation by $\rho_{x^{\prime}, x^{\prime \prime}} \equiv\left\langle x^{\prime}|\rho| x^{\prime \prime}\right\rangle$. We define $r=x^{\prime}-x^{\prime \prime}$ and $R=\left(x^{\prime}+x^{\prime \prime}\right) / 2$ and use supervector Dirac notations, namely, $\rho=\sum_{R, r} \rho(R, r)|R, r\rangle$. The space representation is $\rho(R, r) \equiv \rho_{x^{\prime}, x^{\prime \prime}}$, the momentum representation $\rho(q, P)$ is related by double Fourier transforms, and the intermediate representations are those of Wigner $\rho_{w}(R, P)$ and Bloch $\rho(q ; r)$. For the unitary evolution with $U=\exp [i c t \cos (\boldsymbol{p})]$, the propagator of the Wigner function in momentum representation is

$$
\begin{aligned}
\mathcal{K}\left(q, P \mid q_{0}, P_{0}\right) & =\left\langle P+(q / 2)|U| P_{0}+\left(q_{0} / 2\right)\right\rangle\left\langle P-(q / 2)|U| P_{0}-\left(q_{0} / 2\right)\right\rangle^{*} \\
& =2 \pi \delta\left(q-q_{0}\right) 2 \pi \delta\left(P-P_{0}\right) \exp [-i 2 c t \sin (q / 2) \sin (P)]
\end{aligned}
$$

leading to

$$
\mathcal{K}\left(R, P \mid R_{0}, P_{0}\right)=2 \pi \delta\left(P-P_{0}\right) \int \frac{d q}{2 \pi} \exp \left[-i 2 c t \sin (q / 2) \sin (P)+i q\left(R-R_{0}\right)\right]
$$

Note that this kernel is properly normalized with respect to the integration measure $d R d P /(2 \pi)$.

With $\sin (q / 2) \mapsto(q / 2)$ we get the classical result

$$
\mathcal{K}\left(R, P \mid R_{0}, P_{0}\right)=2 \pi \delta\left(P-P_{0}\right) \delta\left[\left(R-R_{0}\right)-c t \sin (P)\right] .
$$


But quantum mechanically we get

$$
\mathcal{K}\left(R, P \mid R_{0}, P_{0}\right)=\sum_{n} 2 \pi \delta\left(P-P_{0}\right) \delta\left[\left(R-R_{0}\right)-n\right] J_{2 n}[2 c t \sin (P)] .
$$

In the above sum $n$ runs formally over all the integer and half-integer values. Note that Wigner function on a lattice has support on both integer and half-integer lattice points. (Weight on half-integer lattice points is the fingerprint of interference due to superposition of integer lattice locations).

\section{APPENDIX C: THE BLOCH REPRESENTATION}

For an infinite chain the conventional way to define the Bloch representation is to perform $R \mapsto q$ Fourier transform of $\rho(R, r)$ for a given $r$ to obtain $\rho(q ; r)$. Note that $R$ runs over integer values for $r=0,2,4, \ldots$ and over half integer values for $r=1,3,5 \ldots$ This definition has a problem if we consider a finite chain with periodic boundary conditions. Still it can be justified after a short transient if $L$ is large enough because distant points in space loose phase correlation (if there was to begin with). For a small ring (small $L$ ) this might not be the case. Therefore in a previous work [48] we have defined ad hoc the Bloch representation $\rho_{q}(r)$ as the Fourier transform of $\langle x|\rho| x+r\rangle$. The ad hoc definition differs by gauge transformation (and non-intentionally also by sign) from the conventional definition and allows one to correctly handle the periodicity in both coordinates, namely, also in $r$. For a small chain, or for a complete investigation of the eigenvalues problem, these phases are important. See, for example, Ref. [41].

Our system is invariant under translations, and therefore it is natural to perform the diagonalization of $\mathcal{L}$ in the Bloch representation. In practice one can obtain the expressions in Eq. (34) by inspection. As an example, let us see how the expression for $\mathcal{L}^{(c)}$ is obtained. It originates from $i[\cos (\boldsymbol{p}), \rho]$. In the standard representation its matrix elements are

$$
\mathcal{L}^{(c)}\left(x^{\prime}, x^{\prime \prime} \mid x_{0}^{\prime}, x_{0}^{\prime \prime}\right)=i\left\langle x^{\prime}|\cos (\boldsymbol{p})| x_{0}^{\prime}\right\rangle \delta\left(x^{\prime \prime}-x_{0}^{\prime \prime}\right)-i \delta\left(x^{\prime}-x_{0}^{\prime}\right)\left\langle x^{\prime \prime}|\cos (\boldsymbol{p})| x_{0}^{\prime \prime}\right\rangle .
$$

Recall that $\cos (\boldsymbol{p})$ is the sum of displacement operators $e^{\mp i p}$. In supervector notations the above expression can be written in terms of operators $e^{\mp i(1 / 2) q}$ and $e^{\mp i P}$ that induce translations in $R$ and in $r$, respectively. Namely,

$$
\mathcal{L}^{(c)}\left(R, r \mid R_{0}, r_{0}\right)=i\left\langle R, r\left|\cos \left(\frac{\boldsymbol{q}}{2}+\boldsymbol{P}\right)\right| R_{0}, r_{0}\right\rangle-i\left\langle R, r\left|\cos \left(\frac{\boldsymbol{q}}{2}-\boldsymbol{P}\right)\right| R_{0}, r_{0}\right\rangle .
$$

Thus we can write

$$
\mathcal{L}^{(c)}=-i 2 \sin \left(\frac{\boldsymbol{q}}{2}\right) \sin (\boldsymbol{P})=\sin \left(\frac{\boldsymbol{q}}{2}\right)\left[\mathcal{D}_{\perp}-\mathcal{D}_{\perp}^{\dagger}\right] .
$$

In the Bloch $(q, r)$ representation, this superoperator becomes block diagonal in $q$.

\section{APPENDIX D: FROM BLOCH TO WIGNER AND BACK}

In the main text we present in Eq. (34) the Bloch representation $\mathcal{L}^{(q)}$ of the dissipators. The transformation to the Wigner representation is essentially a Fourier transform:

$$
\mathcal{L}\left(R, P \mid R_{0}, P_{0}\right)=\int \frac{d q}{2 \pi} e^{i q\left(R-R_{0}\right)} \iint d r d r_{0} e^{-i r P+i r_{0} P_{0}}\left\langle r\left|\mathcal{L}^{(q)}\right| r_{0}\right\rangle .
$$

Note that the inner integral transforms $\left\langle r\left|\mathcal{L}^{(q)}\right| r_{0}\right\rangle$ to the momentum representation $\left\langle P\left|\mathcal{L}^{(q)}\right| P_{0}\right\rangle$. Note also that $\mathcal{W}\left(P \mid P_{0}\right)=\left\langle P\left|\mathcal{L}^{(q=0)}\right| P_{0}\right\rangle$ are the Fermi golden rule (FGR) transition rates between momentum eigenstates. Commonly the FGR is considered as an approximation, while we have rigorously established that $\mathcal{W}\left(P \mid P_{0}\right)$ can be used within an effective rate equation in order to evaluate the exact quantum result for $D$.

In the main text we use a discrete momentum notation such that $\langle r \mid P\rangle=L^{-1 / 2} \exp (i P R)$, etc. Consequently, in the discrete version of Eq. (D1), the integrand of the $d r d r_{0}$ integral contains an extra $1 / L$ factor. On the other hand, for summation $\sum_{P}$ over momenta the measure becomes $[L /(2 \pi)] d P$.

\section{Transforming to Bloch}

It is convenient to handle the calculations of the spectrum on equal footing for all the coupling schemes, for both Ohmic and Boltzmann versions of the dissipators. For this purpose we have to transform Eq. (42) back from the Wigner representation to the Bloch representation using Eq. (43). Note that this equation does not depend on $q$, reflecting the $\delta\left(R-R_{0}\right)$ of the transitions. Making the distinction between the diagonal terms $\left(P=P_{0}\right)$ and the off-diagonal terms $\left(P \neq P_{0}\right)$, taking into account that by definition the kernel conserves probability, namely, $\sum_{P} \mathcal{W}\left(P \mid P_{0}\right)=0$, one can write

$$
\left\langle r\left|\mathcal{L}^{(q)}\right| r_{0}\right\rangle=\tilde{\mathcal{W}}\left(r, r_{0}\right)-\tilde{\mathcal{W}}\left(0, r_{0}-r\right),
$$

where

$$
\tilde{\mathcal{W}}\left(r, r_{0}\right)=\frac{1}{L} \iint \mathcal{W}\left(P \mid P_{0}\right) e^{i P r-i P_{0} r_{0}} \frac{L}{2 \pi} d P \frac{L}{2 \pi} d P_{0} .
$$


In the latter expression it is implicit that $P=P_{0}$ has measure zero, so it reflects the contribution of the $P \neq P_{0}$ terms in the discrete sum of Eq. (43). Finally, note that for $\eta=0$ the Bloch kernel is diagonal (the nonzero elements are those with $r=r_{0}$ ) and that from Eq. (41) it follows that for $r=r_{0}= \pm 1$ we have by convention $\left\langle r\left|\mathcal{L}^{(q)}\right| r_{0}\right\rangle=-(v / 2)$.

\section{General kernel}

We consider a kernel $\mathcal{W}\left(P \mid P_{0}\right)$ of width $w=2 \pi / \ell$. Its normalization $C$ should be determined such that the $d p$ integral over $[1-\cos (p)]$ equals $1 / 2$ (see the last sentence of the previous paragraph). Note this normalization affects the result for $C_{\|}$but not the significant result for $A_{\|}$. Expressing the double-integral Eq. (D3) with $k=\left(P+P_{0}\right) / 2$ and $p=P-P_{0}$, and using the notation $z=(c / T)$, it reads

$$
\tilde{\mathcal{W}}\left(r, r_{0}\right)=v \int_{-w / 2}^{w / 2} d p C e^{(i p / 2)\left(r+r_{0}\right)} \int_{-\pi}^{\pi} \frac{d k}{2 \pi} e^{i k\left(r-r_{0}\right)} \exp [-z \sin (p / 2) \sin (k)] .
$$

The inner integral may be written as $\mathrm{I}_{r-r_{0}}[-z \sin (p / 2)]$; however, to calculate $A_{\|}$in the high-temperature limit it is enough to Taylor expand $z$ to second order. The inner integral provides "selection rules." The zero-order result gives a constant along the main diagonal of $\tilde{\mathcal{W}}$, while the first order contributes to the near-neighbor hopping $\left(\left|r-r_{0}\right|=1\right)$. The second order contributes both to next-near-neighbor hopping and to the main diagonal.

Including in $\mathcal{L}^{(q)}$ also the $c \mathcal{L}^{(c)}$ term, and using the method described in Sec. VII B, one finds

$$
A_{\|}(w)=\frac{w^{2}+16 \sin ^{2}\left(\frac{w}{2}\right)-\sin ^{2}(w)+4 w \sin (w)-4 \sin \left(\frac{w}{2}\right)[3 w+\sin (w)]}{16\left[w-2 \sin \left(\frac{w}{2}\right)\right][w-\sin (w)]}-\frac{3 w-8 \sin \left(\frac{w}{2}\right)+\sin (w)}{32\left[w-2 \sin \left(\frac{w}{2}\right)\right]} .
$$

The first term is the Ohmic result, while the second term is added to get the Boltzmann-corrected result. The results for the X coupling and for the S coupling in Eq. (13) are obtained for $w=0$ and $w=2 \pi$, respectively.

\section{Boltzmann case for $S / B$}

For the Boltzmann-corrected versions of $S$ and $B$ one obtains

$$
\begin{gathered}
\tilde{\mathcal{W}}^{(S)}\left(r, r_{0}\right)=v_{S} \mathrm{I}_{r}(z / 2) \mathrm{I}_{r_{0}}(-z / 2), \\
\tilde{\mathcal{W}}^{(B)}\left(r, r_{0}\right)=2 v_{B} \mathrm{I}_{r}(z / 2) \mathrm{I}_{r_{0}}(-z / 2)+v\left[\mathrm{I}_{r+1}(z / 2) \mathrm{I}_{r_{0}-1}(-z / 2)+\mathrm{I}_{r-1}(z / 2) \mathrm{I}_{r_{0}+1}(-z / 2)\right] .
\end{gathered}
$$

Expanding in $z=(c / T)$, the first-order result for $\mathcal{L}^{(q)}$ is a $q=0$ version of the $\mathrm{S} / \mathrm{B}$ dissipators that were presented in the main text Eq. (34). In the Boltzmann-corrected approximation, both schemes acquire second-order terms $-(3 / 16)(c / T)^{2} v| \pm 1\rangle\langle \pm 1|$ that are required for the calculation of $D$. For the $S$ coupling scheme one finds additional second-order terms that are needed for the calculations, namely, $-(3 / 32)(c / T)^{2} v|1\rangle\langle-1|$ and $-(3 / 32)(c / T)^{2} v|-1\rangle\langle 1|$.

[1] A. O. Caldeira and A. J. Leggett, Path integral approach to quantum Brownian motion, Physica A 121, 587 (1983).

[2] A. O. Caldeira and A. J. Leggett, Quantum tunneling in a dissipative system, Ann. Phys. 149, 374 (1983).

[3] V. Hakim and V. Ambegaokar, Quantum theory of a free particle interacting with a linearly dissipative environment, Phys. Rev. A 32, 423 (1985).

[4] A. Madhukar and W. Post, Exact Solution for the Diffusion of a Particle in a Medium with Site Diagonal and Off-Diagonal Dynamic Disorder, Phys. Rev. Lett. 39, 1424 (1977).

[5] U. Weiss and H. Grabert, Quantum diffusion of a particle in a periodic potential with ohmic dissipation, Phys. Lett. A 108, 63 (1985).

[6] N. Kumar and A. M. Jayannavar, Quantum diffusion in thin disordered wires, Phys. Rev. B 32, 3345 (1985).

[7] C. Aslangul, N. Pottier, and D. Saint-James, Quantum ohmic dissipation: Cross-over between quantum tunneling and thermally resisted motion in a biased tight-binding lattice, J. Phys. 47, 1671 (1986).

[8] U. Weiss, M. Sassetti, T. Negele, and M. Wollensak, Dissipative quantum dynamics in a multiwell system, Z. Phys. B 84, 471 (1991).
[9] D. Roy, Crossover from ballistic to diffusive thermal transport in quantum Langevin dynamics study of a harmonic chain connected to self-consistent reservoirs, Phys. Rev. E 77, 062102 (2008).

[10] A. Amir, Y. Lahini, and H. B. Perets, Classical diffusion of a quantum particle in a noisy environment, Phys. Rev. E 79, 050105(R) (2009).

[11] S. Lloyd, M. Mohseni, A. Shabani, and H. Rabitz, The quantum goldilocks effect: on the convergence of timescales in quantum transport, arXiv:1111.4982.

[12] J. M. Moix, M. Khasin, and J. Cao, Coherent quantum transport in disordered systems: I. The influence of dephasing on the transport properties and absorption spectra on one-dimensional systems, New J. Phys. 15, 085010 (2013).

[13] J. Wu, R. J. Silbey, and J. Cao, Generic Mechanism of Optimal Energy Transfer Efficiency: A Scaling Theory of the Mean First-Passage Time in Exciton Systems, Phys. Rev. Lett. 110, 200402 (2013).

[14] Y. Zhang, G. L. Celardo, F. Borgonovi, and L. Kaplan, Openingassisted coherent transport in the semiclassical regime, Phys. Rev. E 95, 022122 (2017). 
[15] Y. Zhang, G. L. Celardo, F. Borgonovi, and L. Kaplan, Optimal dephasing for ballistic energy transfer in disordered linear chains, Phys. Rev. E 96, 052103 (2017).

[16] T. Dekorsy, A. Bartels, H. Kurz, K. Köhler, R. Hey, and K. Ploog, Coupled Bloch-Phonon Oscillations in Semiconductor Superlattices, Phys. Rev. Lett. 85, 1080 (2000).

[17] F. Dubin, R. Melet, T. Barisien, R. Grousson, L. Legrand, M. Schott, and V. Voliotis, Macroscopic coherence of a single exciton state in an organic quantum wire, Nat. Phys. 2, 32 (2006).

[18] T. R. Nelson, D. Ondarse-Alvarez, N. Oldani, B. RodriguezHernandez, L. Alfonso-Hernandez, J. F. Galindo, V. D. Kleiman, S. Fernandez-Alberti, A. E. Roitberg, and S. Tretiak, Coherent exciton-vibrational dynamics and energy transfer in conjugated organics, Nat. Commun. 9, 2316 (2018).

[19] A. Schmid, Diffusion and Localization in a Dissipative Quantum System, Phys. Rev. Lett. 51, 1506 (1983).

[20] M. P. A. Fisher and W. Zwerger, Quantum Brownian motion in a periodic potential, Phys. Rev. B 32, 6190 (1985).

[21] C. Aslangul, N. Pottier, and D. Saint-James, Quantum Brownian motion in a periodic potential: A pedestrian approach, J. Phys. (France) 48, 1093 (1987).

[22] G. S. Engel, T. R. Calhoun, E. L. Read, T.-K. Ahn, T. Mančal, Y.-C. Cheng, R. E. Blankenship, and G. R. Fleming, Evidence for wavelike energy transfer through quantum coherence in photosynthetic systems, Nature (London) 446, 782 (2007).

[23] H. van Amerongen, R. van Grondelle, and L. Valkunas, Photosynthetic Excitons (World Scientific, Singapore, 2000).

[24] T. Ritz, A. Damjanović, and K. Schulten, The quantum physics of photosynthesis, Chem. Phys. Chem. 3, 243 (2002).

[25] Y.-C. Cheng and G. R. Fleming, Dynamics of light harvesting in photosynthesis, Annu. Rev. Phys. Chem. 60, 241 (2009).

[26] M. B. Plenio and S. F. Huelga, Dephasing-assisted transport: Quantum networks and biomolecules, New J. Phys. 10, 113019 (2008).

[27] P. Rebentrost, M. Mohseni, I. Kassal, S. Lloyd, and A. AspuruGuzik, Environment-assisted quantum transport, New J. Phys. 11, 033003 (2009).

[28] P. Rebentrost, M. Mohseni, and A. Aspuru-Guzik, Role of quantum coherence and environmental fluctuations in chromophoric energy transport, J. Phys. Chem. B 113, 9942 (2009).

[29] M. Sarovar and K. B. Whaley, Design principles and fundamental trade-offs in biomimetic light harvesting, New J. Phys. 15, 013030 (2013).

[30] K. Higgins, S. Benjamin, T. Stace, G. Milburn, B. W. Lovett, and E. Gauger, Superabsorption of light via quantum engineering, Nat. Commun. 5, 4705 (2014).

[31] G. L. Celardo, F. Borgonovi, M. Merkli, V. I. Tsifrinovich, and G. P. Berman, Superradiance transition in photosynthetic lightharvesting complexes, J. Phys. Chem. C 116, 22105 (2012).

[32] H. Park, N. Heldman, P. Rebentrost, L. Abbondanza, A. Iagatti, A. Alessi, B. Patrizi, M. Salvalaggio, L. Bussotti, M. Mohseni et al., Enhanced energy transport in genetically engineered excitonic networks, Nat. Mater. 15, 211 (2016).

[33] V. Tiwari, W. K. Peters, and D. M. Jonas, Electronic resonance with anticorrelated pigment vibrations drives photosynthetic energy transfer outside the adiabatic framework, Proc. Nat. Acad. Sci. U.S.A. 110, 1203 (2013).
[34] R. Tempelaar, T. L. C. Jansen, and J. Knoester, Vibrational beatings conceal evidence of electronic coherence in the FMO light-harvesting complex, J. Phys. Chem. B 118, 12865 (2014).

[35] H.-G. Duan, V. I. Prokhorenko, R. J. Cogdell, K. Ashraf, A. L. Stevens, M. Thorwart, and R. J. D. Miller, Nature does not rely on long-lived electronic quantum coherence for photosynthetic energy transfer, Proc. Nat. Acad. Sci. U.S.A. 114, 8493 (2017).

[36] M. Maiuri, E. E. Ostroumov, R. G. Saer, R. E. Blankenship, and G. D. Scholes, Coherent wavepackets in the Fenna-MatthewsOlson complex are robust to excitonic-structure perturbations caused by mutagenesis, Nat. Chem. 10, 177 (2018).

[37] E. Thyrhaug, R. Tempelaar, M. J. P. Alcocer, K. Žídek, D. Bína, J. Knoester, T. L. C. Jansen, and D. Zigmantas, Identification and characterization of diverse coherences in the Fenna-Matthews-Olson complex, Nat. Chem. 10, 780 (2018).

[38] J. Cao, R. J. Cogdell, D. F. Coker, H.-G. Duan, J. Hauer, U. Kleinekathöfer, T. L. C. Jansen, T. Mančal, R. J. D. Miller, J. P. Ogilvie, V. I. Prokhorenko, T. Renger, H.-S. Tan, R. Tempelaar, M. Thorwart, E. Thyrhaug, S. Westenhoff, and D. Zigmantas, Quantum biology revisited, Sci. Adv. 6, eaaz4888 (2020).

[39] D. Cohen, Quantum Dissipation Versus Classical Dissipation for Generalized Brownian Motion, Phys. Rev. Lett. 78, 2878 (1997).

[40] D. Cohen, Unified model for the study of diffusion localization and dissipation, Phys. Rev. E 55, 1422 (1997).

[41] M. Esposito and P. Gaspard, Emergence of diffusion in finite quantum systems, J. Stat. Phys. 121, 463 (2005).

[42] S. Wang, Generalization of the Thomas-Reiche-Kuhn and the Bethe sum rules, Phys. Rev. A 60, 262 (1999).

[43] D. Cohen, Quantum Dissipation Due to the Interaction with Chaotic Degrees of Freedom and the Correspondence Principle, Phys. Rev. Lett. 82, 4951 (1999).

[44] A. Stotland and D. Cohen, Diffractive energy spreading and its semiclassical limit, J. Phys. A: Math. Gen. 39, 10703 (2006).

[45] T. Hartmann, F. Keck, H. J. Korsch, and S. Mossmann, Dynamics of Bloch oscillations, New J. Phys. 6, 2 (2004).

[46] H.-P. Breuer and F. Petruccione, The Theory of Open Quantum Systems (Oxford University Press on Demand, Oxford, 2002).

[47] A. J. Leggett, S. Chakravarty, A. T. Dorsey, M. P. A. Fisher, A. Garg, and W. Zwerger, Dynamics of the dissipative two-state system, Rev. Mod. Phys. 59, 1 (1987).

[48] D. Shapira and D. Cohen, Quantum stochastic transport along chains, Sci. Rep. 10, 10353 (2020).

[49] D. Cohen, Lecture notes in statistical mechanics and mesoscopics, arXiv:1107.0568.

[50] A. Gärtner, A. W. Holleitner, J. P. Kotthaus, and D. Schuh, Drift mobility of long-living excitons in coupled GaAs quantum wells, Appl. Phys. Lett. 89, 052108 (2006).

[51] N. Karl, Charge carrier transport in organic semiconductors, Synth. Met. 133-134, 649 (2003), Proceedings of the Yamada Conference LVI. The Fourth International Symposium on Crystalline Organic Metals, Superconductors and Ferromagnets (ISCOM 2001).

[52] D. Shapira and D. Cohen, Lognormal-like statistics of a stochastic squeeze process, Phys. Rev. E 96, 042152 (2017). 